\title{
Persistent dynamic correlations in self-organized critical systems away from their critical point
}

\author{
Ryan Woodard ${ }^{\mathrm{a}, *, 1}$, David E. Newman ${ }^{\mathrm{a}}$, Raúl Sánchez ${ }^{\mathrm{b}, 2}$, Benjamin A. Carreras \\ ${ }^{a}$ University of Alaska Fairbanks, Fairbanks, Alaska 99775-5920, USA \\ ${ }^{\mathrm{b}}$ Universidad Carlos III de Madrid, 28911 Leganés, Madrid, Spain \\ ${ }^{\mathrm{c}}$ Oak Ridge National Laboratory, Oak Ridge, Tennessee 37831-8070, USA
}

\begin{abstract}
We show that correlated dynamics and long time memory persist in self-organized criticality (SOC) systems even when forced away from the defined critical point that exists at vanishing drive strength. These temporal correlations are found for all levels of external forcing as long as the system is not overdriven. They arise from the same physical mechanism that produces the temporal correlations found at the vanishing drive limit, namely the memory of past events stored in the system profile. The existence of these correlations contradicts the notion that a SOC time series is simply a random superposition of events with sizes distributed as a power law, as has been suggested by previous studies.
\end{abstract}

Keywords: Self-organized criticality; Running sandpile; Correlations; Hurst; Pulses

\section{Introduction}

Are events in a self-organized criticality (SOC) [1,2] system random or correlated? This is a still ongoing and important topic of discussion in the SOC community that has been argued from both sides to propose or invalidate practical tests for SOC behavior [3-11]. One specific point of this dialogue concerns the distribution of the amount of time between the initiation of successive events (waiting, laminar or first return times) in a system suspected of exhibiting SOC behavior. At issue is whether or not the probability density function (PDF) of this measure should always scale exponentially or as a power law for a SOC system. Three recent examples of this are drawn from space plasma physics [3], laboratory confined fusion plasma physics [4] and earthquake geophysics [9]. The authors of all of these studies first assume that events in a SOC system must be uncorrelated. They then report observations of waiting times distributed as power laws and draw separate but similar conclusions that the systems' (space plasma, fusion plasma, earthquakes) dynamics cannot be consistent with SOC.

\footnotetext{
${ }^{*}$ Corresponding author.

E-mail address: rywo@bas.ac.uk (R. Woodard).

${ }^{1}$ Present address: British Antarctic Survey, Madingley Road, Cambridge, CB3 0ET, UK.

${ }^{2}$ Present address: Oak Ridge National Laboratory, Oak Ridge, Tennessee 37831-8070, USA.
} 
The root of this controversy is to be found in the misconceptions that "strong correlations between successive [events is] at variance with the SOC model" [3] and that an event "can occur randomly anywhere at any time and...cannot 'know' how large it will become" [7,9]. Based on these statements, the idea of exponentially distributed waiting times could be easily derived [3]. But it has been known for some time that these notions simply do not hold in a SOC system [5,12]. They were probably encouraged, in the early days of SOC, by analytical calculations $[13,14]$ that appeared to reproduce quite well the power spectrum of the avalanche activity observed in the Bak-Tang-Wiesenfeld (BTW) sandpile [1] (and later in the directed running sandpile [15]) when modeling it as a random superposition of events with power-law distributed sizes. Since then, a state of confusion that still persists appears to surround this issue, at least among those looking for SOC features in physical systems, with quite a few papers accepting $[3,4,9,11]$ and others denying $[5,8,10,16]$ the validity of these notions.

In this paper, we show explicitly that features exist in the power spectra of SOC systems that signify dynamical correlations and that, therefore, any assumption or test based on the absence of temporal correlations is meaningless. By "dynamical" we mean that temporal correlations are caused by the system dynamics, not by a hypothetical non-randomness in the external drive [5]. To study them, we have examined the avalanche activity time series of the same one-dimensional running sandpile model analyzed in Ref. [15] as our prototypical SOC model. The benefit of using this model is that it allows us to analyze systems with a finite drive strength instead of just the vanishing drive limit in which the absorbing phase transition responsible for the appearance of criticality and universal behavior was identified [17-20]. Indeed, in many practical applications a finite drive is more appropriate. This is the case, for instance, in most magnetized plasmas, either inside the Sun [21] or confined in an earth-based tokamak [22].

Taking advantage of increased computer power over that available at the time of Ref. [15] we explore timescales up to four decades longer than those used in that study for varying driving rates and system sizes. Because of this, dynamical correlations not previously detected in the power spectrum of the avalanche activity time series (associated with $f^{-\beta}$ power-law regions of exponent $0<\beta \leqslant 1$ ) are now apparent. The exponent is found to increase with the drive strength, reaching $\beta=1$ only at the higher drives. We show that this is not a signature of the increasing dominance of avalanche overlapping (as stated previously in Ref. [15]), but just a consequence of the fact that the timescales where the correlations are present are also displaced towards higher frequencies at stronger drives. Therefore, avalanche overlapping is not the cause of the $1 / f$ region seen in the directed running sandpile.

The changing value of $\beta$ might seem to suggest that the character of the correlations is changing with the drive as we move away from the critical point. However, when analyzing the same activity signals with rescaled range $(R / S)$ analysis $[23,24]$ to estimate the Hurst exponent $H$, a very different conclusion is drawn. Dynamical correlations are associated with $H>0.5$. We find a constant Hurst exponent $H \simeq 0.8$ for all values of driving rate over the same timescales described above, where $\beta>0$ in the power spectrum. $H$ remains constant as $\beta$ changes from $\beta \simeq 0.4$ at the lowest driving rate studied here to $\beta \simeq 1$ at the highest driving rates. Interestingly, in the vanishing drive limit [25] we find the same value of $H$. In this natural limit of the running sandpile model, avalanches do not overlap, by definition, because the external drive is turned off as soon as an event is initiated. The origin of the correlations must thus be found, then, in a mechanism other than avalanche overlap. Specifically, the origin of temporal correlations in the sandpile model is the shape of the system profiles, carved by past avalanches, where the memory of the system history is stored.

In this report, we do not set out to prove that temporal correlations exist in a SOC system. In the words of a helpful referee, "there is no question whether correlations exist in self-organized or any other critical systems. They do, basically by definition." Such correlations can be proved through the existence of diverging correlation lengths and times [26]. Given that temporal correlations exist in SOC systems, our goals are to lay to rest the controversy mentioned in the first paragraph and to address the questions: How does one identify temporal correlations in a real system? What measures should one use? What do those measures say? What mechanism(s) produce(s) the correlations? We use the power spectrum and $R / S$ analysis in this study but do not claim that they are the only possible applicable measures. Multiple measures should be used for completely thorough studies of dynamical systems.

The paper is organized as follows: in Section 2 the basics of the running sandpile and analysis techniques are reviewed. Numerical results obtained for the avalanche activity time series are described in Section 3 and discussed in Section 4. Finally, some conclusions are drawn in Section 6. 


\section{Model and measures}

\subsection{Model}

The running sandpile can be described by four parameters: $L, P_{0}, Z_{\text {crit }}$ and $N_{\mathrm{f}}$. Consider a single column of $L$ cells. Each cell contains an integer number of "sand grains"; this number is the height of the cell. Sand is added to each cell by a random "rain" from above. That is, at each time step for each cell, there is a probability $0<P_{0}<1$ that a grain of sand will be added to it. The average input current into the entire system is $\overline{J_{\mathrm{IN}}}=P_{0} L$ grains per time step. The local gradient $Z_{\mathrm{n}}$ is the difference in height between two neighboring cells. If this local gradient exceeds a critical gradient $Z_{\text {crit }}$ then an avalanche occurs. An avalanche stabilizes the local gradient by transferring $N_{\mathrm{f}}$ grains of sand from the higher cell to the lower in a single event called a flip [25] or a toppling (the more standard term). This avalanche can make $Z_{\mathrm{n}+1}$ and/or $Z_{\mathrm{n}-1}$ unstable at the next time step so that the avalanche spreads to other cells. In this way, spatially and temporally extended events in a system can occur. This sandpile can be extended to two or more dimensions [2] but we will only discuss the one-dimensional case. For this study, we want to explore the effects of changing system size $(L)$ and external driving rate (probability $P_{0}$ ) so we have arbitrarily fixed the parameters $Z_{\text {crit }}=8$ and $N_{\mathrm{f}}=3$. In Ref. [22] it was shown that the system dynamics does not qualitatively change for different values of $Z_{\text {crit }}$ and $N_{\mathrm{f}}$, as long as $1<N_{\mathrm{f}}<Z_{\text {crit }} / 2$.

Note that time is well-defined in this running sandpile model as opposed to the vanishing drive model (the original BTW model, for instance) where external forcing (sand addition) is suspended as long as there is activity in the system. In this running sandpile, sand can be added at each cell at every time step with a probability $P_{0}$ even if an avalanche is active. When we increase the level of external forcing by increasing $P_{0}$, we increase the probability of a grain of sand being added to each cell and therefore increase the probability of an avalanche. But we do not change the time step-the clock continues to tick regularly regardless of system activity. Computationally, this requires simultaneous random adding of grains (forcing) and checking for instability (relaxation) within the same programming loop. A natural time scale for the system, then, can be thought of as $P_{0}^{-1}$.

The time series that we analyze is the instantaneous total avalanche activity or, in short, the activity $\rho_{a}$, which is defined as the total number of unstable sites (where $Z \geqslant Z_{\text {crit }}$ ) at each time step. An unstable cell transports $N_{\mathrm{f}}$ grains of sand to the next cell. The activity at each time step can be thought of as the instantaneous (potential) energy dissipation in the system or the total instantaneous flux through it. Avalanches in a $\rho_{a}$ time series appear as structures - sequential non-zero activity separated by periods of inactivity, or quiet times. We analyze activity data for times after steady state has been reached, which is when the long time average number of topplings approaches a constant value.

Two temporal measures from the activity series are discussed in this paper: durations and quiet times. A duration is the number of time steps during which the system is active; we refer to this as an avalanche. Note that for the running sandpile that we use in this study, an avalanche defined in this way could actually comprise two or more distinct avalanches that happen to be active at the same time in the system. At highdrive, this tends to be the case but at low-drive individual avalanches can be distinguished through analysis of the pulse shape in the activity time series. A quiet time is defined as the length of an uninterrupted sequence of zero topplings (inactivity) between two avalanches. It is the length of time that the system is in an absorbing state. In the randomly driven sandpile, probability density functions (PDFs) of unadulterated avalanche durations scale as a power law in the self-similar range [27] while the PDFs of quiet times scale exponentially $[5,6]$.

An important part of this study is to quantify the effect of changing the drive strength and system size on correlations. First, it is important to note that for critical dynamics to exist, the system must not only be underdriven (i.e., the flux in, $\overline{J_{\mathrm{IN}}}=P_{0} L$, must never exceed the maximum possible flux out of the bottom cell, $\overline{J_{\text {OUT }}}=N_{\mathrm{f}}$ ), but it must also satisfy $\overline{J_{\text {IN }}}<N_{\mathrm{f}} / 2$, so that a cell can alternate between stable and unstable until the avalanche ends or washes past the cell [22]. Second, note that the difference between driving close/far from the condition $\overline{J_{\mathrm{IN}}}<N_{\mathrm{f}} / 2$ is to increase/decrease the degree of avalanche overlapping. That is, if $\overline{J_{\mathrm{IN}}} \ll N_{\mathrm{f}} / 2$ then there will be very few cases of simultaneous avalanches and hence very little avalanche overlapping. In practice, $\overline{J_{\mathrm{IN}}}$ can be set as small as desired to effectively eliminate all overlapping, thus allowing identification 

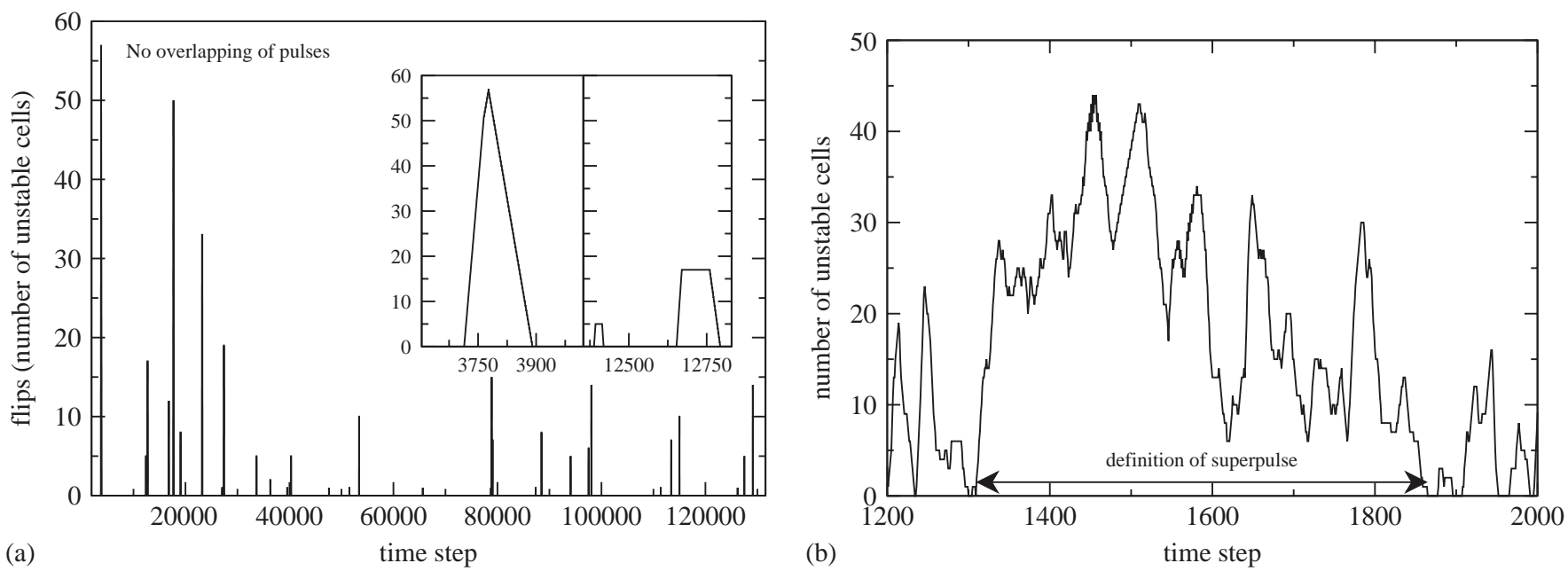

Fig. 1. Partial activity time series from running sandpile model at steady state for (a) low and (b) high-drive. At low-drive $\left(P_{0} L^{2}=0.2\right)$, there are approximately 30 distinct and separate events in $\sim 10^{5}$ time steps while at high-drive $\left(P_{0} L^{2}=100\right)$ there are far more events and their identification is made difficult by almost constant overlapping. Note the trapezoid shape of a single, distinct avalanche in the magnified inset of the low-drive case. A superpulse is defined for high-drive as the sequence of activity between consecutive quiet times.

and analysis of single avalanches. In this limit, avalanche dynamics and statistics are the same as the vanishing drive (non-running) sandpile of [25].

We want to quantify the amount of temporal event overlapping and we want to compare systems of different sizes $(L)$. If the average avalanche duration $\bar{d}(\sim L)$ is greater than the average trigger time $T_{t}\left(\sim N_{\mathrm{f}} / P_{0} L\right)$ [5] then avalanches will overlap and therefore the condition for overlapping in time is $\bar{d}>T_{t}$ or $P_{0} L^{2}>N_{\mathrm{f}}$. (This is in contrast to the limit for spatial overlap previously given in Eq. (4) in Ref. [15].) For a fixed $N_{\mathrm{f}}$, this condition encompasses both size and drive strength so we define the effective driving rate as $J_{\mathrm{E}}=P_{0} L^{2}$. At steady state, of course, flux into and out of the system are still equal, $\overline{J_{\mathrm{IN}}}=P_{0} L . J_{\mathrm{E}}$ is simply a parameter defined to compare systems of different sizes that have proportional levels of overlapping. In what follows, low-drive will refer to systems where overlapping of events is negligible (i.e., $J_{\mathrm{E}} \ll N_{\mathrm{f}}$ ), and high-drive will refer to cases when overlapping becomes significant (i.e., $J_{\mathrm{E}} \gg N_{\mathrm{f}}$ ) (see Fig. 1). This will be discussed further in Section 5.

\subsection{Measures}

Though a variety of measures can be used, our tools to detect correlations are power spectra and rescaled range $(R / S)$ analysis. The discrete power spectrum of a data set $X(t)$ is defined as the square of the discrete Fourier transform, $S(f)=|F(f)|^{2}$, where

$$
F(f)=N^{-1} \sum_{t=0}^{N-1} X(t) \mathrm{e}^{-\mathrm{i} 2 \pi(f / N) t}
$$

What does the power spectrum say about correlations in a series? More appropriate here, what does a power spectrum that scales as a power law $f^{-\beta}$ have to say about persistence in a series? For $\beta>0$, the low frequencies are more important in the dynamics so the series tends to look relatively smooth and trends persist. Such series are said show persistence. For $\beta<0$, the high-frequency components are more important and the series is very rough. Trends do not persist for long and the series is called antipersistent. Truly random noise (white noise) has a spectrum that scales with $\beta=0$ [28].

The Hurst exponent, $H \in[0,1][23]$, can be used to measure persistence in a time series. A value of $H>0.5$ implies persistence, $H<0.5$ antipersistence and $H=0.5$ uncorrelated. We measure $H$ by using $R / S$ analysis [23,29,24]. The rescaled range is defined as $R^{\prime}(\tau) \equiv R(\tau) / S(\tau)$, where $S(\tau)$ is the standard 
deviation and

$$
\begin{aligned}
& R(\tau)=\max _{1 \leqslant k \leqslant \tau} W(k, \tau)-\min _{1 \leqslant k \leqslant \tau} W(k, \tau) \quad \text { (range), } \\
& W(k, \tau)=\sum_{t=1}^{k}\left(X_{t}-\langle X\rangle_{\tau}\right) \quad \text { (cumulative deviation) and } \\
& \langle X\rangle_{\tau}=\frac{1}{\tau} \sum_{t=1}^{\tau} X_{t} \text { (mean). }
\end{aligned}
$$

If the rescaled range of the time series exhibits regions that scale as $R^{\prime}(\tau) \sim \tau^{H}$, then $H$ is the Hurst exponent.

Need to work on this paragraph: In some cases, $H$ as estimated by $R / S$ analysis can be related to $\beta$. For instance, for a time series of stationary independent identically distributed random variables (drawn from a Gaussian distribution), $\beta=2 H-1[30]$. If, however, the random variables are instead distributed with a fat tail that scales as $\sim x^{-\mu}$ then the two parameters are more generally related as $\beta=2\left(H+1-\mu^{-1}\right)$ [24]. In both examples, a crucial underlying assumption is that the random variables that comprise the time series are stable and stationary. This should be kept in mind for Section 4.3 below, where we show and discuss the fact that $\beta$ and $H$ are not simply related for the activity of the running sandpile.

\section{Results}

In this section, we describe the primary qualitative features of the power spectra and $R / S$ analysis of activity time series obtained for different driving rates and system sizes. The main theme is that distinct powerlaw regions always exist in both measures; we defer the interpretations of the regions to the following section.

The power spectra of the activity look very different depending upon whether the driving rate is low or high. Fig. 2 shows the spectra for over five orders of magnitude of effective driving rate, $J_{\mathrm{E}}=P_{0} L^{2}$, which increases from top to bottom in the figure. [The sandpile size is $L=200$, but very similar results have been obtained for sizes between $L=50$ and 2000.] The lowest drive used is $P_{0} L^{2}=0.002$ and the highest is $P_{0} L^{2}=296$, chosen to stay below the normal overdrive limit of $P_{0} L<N_{\mathrm{f}} / 2$, discussed above. Multiple distinct power law regions are seen in all of the spectra. The highest frequency regions share a common slope $(\simeq 3.5)$. At low-drive a very prominent hump at low frequency moves to higher frequency as driving rate increases.

For clarity, selected spectra for a low, a medium and a high-drive case are shown in Fig. 3(a) for $L=200$. For the lowest drive case, six power-law regions can be distinguished, labeled $A-F$ as frequency decreases. They are separated by breakpoints denoted by $T_{k}^{-1}, k$ being the appropriate region label. Similarly, four regions are found in the highest drive case, labeled $A, D, E$ and $F$ for reasons to be discussed in the next section. The regions and the labeling convention are illustrated in the cartoons in Figs. 4(a) and (c). [Fig. 4(c) is based on Fig. 6 of Ref. [15].]

Next, we will describe the $R / S$ analysis results. They are shown, for the same selected cases, in Fig. 3(b). Again, different power-law regions can be distinguished: five distinct regions for the lowest drive case and four for the highest. They are labeled according to the same convention as the power spectra (Figs. 4(b) and (d)), except for the shortest timescale region, labeled instead as " $A / B$ ". Again, relevant breakpoints are identified and labeled.

The labeling chosen for the spectrum and $R / S$ analysis breakpoints is not arbitrary. Breakpoints are compared in Fig. 5(a), where it is found that those of the two measures, found independently, agree very closely with each other. [The $R / S$ breakpoints appear at slightly longer timescales than those of the power spectrum, but this effect is known from comparisons with Hurst exponents determined via the structure functions method [33,34].] We conclude that both measures distinguish the same dynamical regions through the identification of different power-law regions.

For the discussion in the next section it is interesting to know how these breakpoints scale with driving rate (see Fig. 5(b)). Two different behaviors can be distinguished: the breakpoints associated with the $A$ and $B$ (and $A / B$ ) regions are independent of the drive, while all of the others scale (almost) linearly with it, moving to higher frequencies (or shorter time lags for the $R / S$ analysis) as the drive increases. 


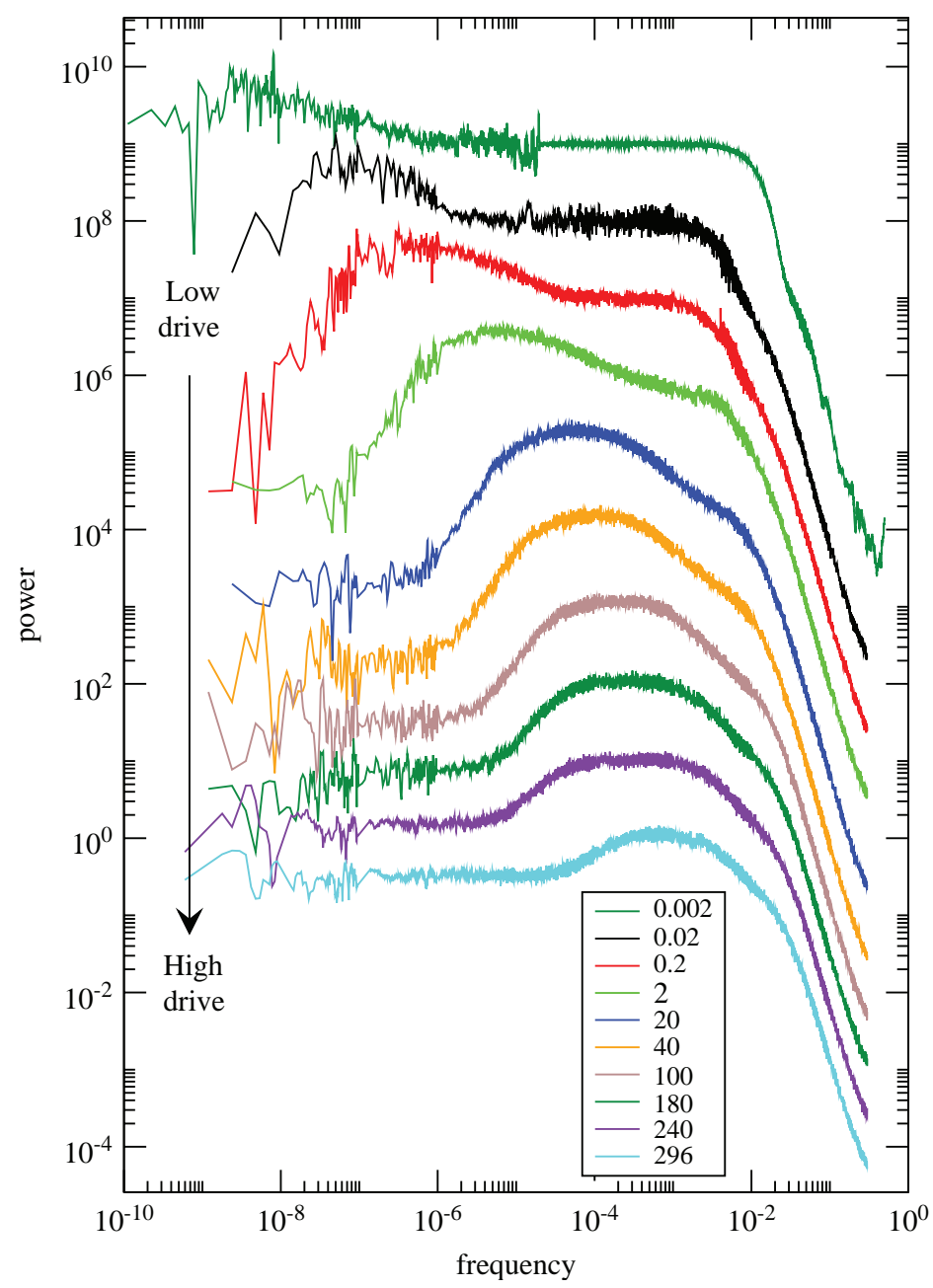

Fig. 2. (Color online) Power spectra of activity time series of $L=200$ sandpile for five orders of magnitude of effective driving rate in $P_{0} L^{2} \in[0.002,296]$. Spectra have been shifted along the $y$ axis for easier viewing.
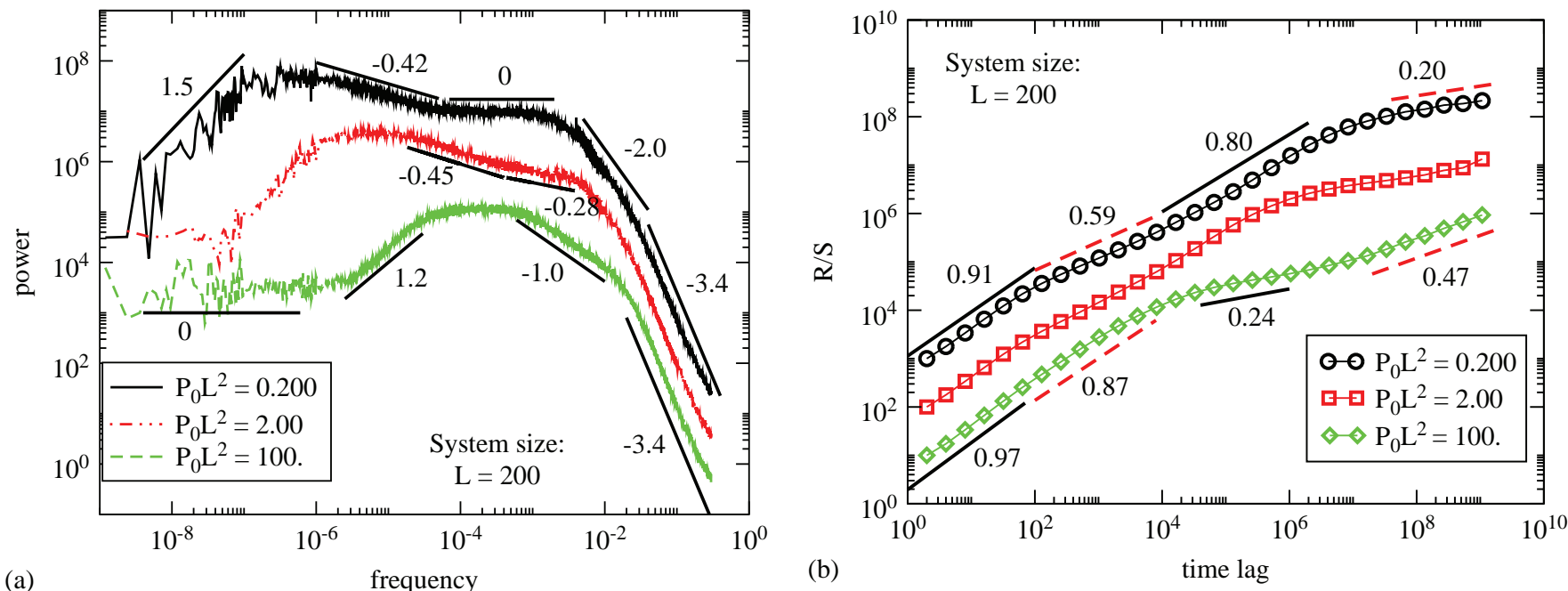

Fig. 3. (Color online) (a) Power spectra and (b) $R / S$ analysis of activity for fixed system size and low, medium and high driving rates. The $y$ values of both measures have been shifted for easier viewing. In the spectra $(R / S)$, six (five) regions of low-drive and four regions of highdrive are shown by solid lines. Lines are power laws; numbers show values of $\beta$ in spectra and $H$ in $R / S$. Lowest frequency $f^{0}$ region of spectra and $H=0.5$ of $R / S$ for the low-drive case is not seen because of the finite size of the time series. Its existence is assumed based on the $f^{0}$ regions seen in the spectra of higher drive cases. 


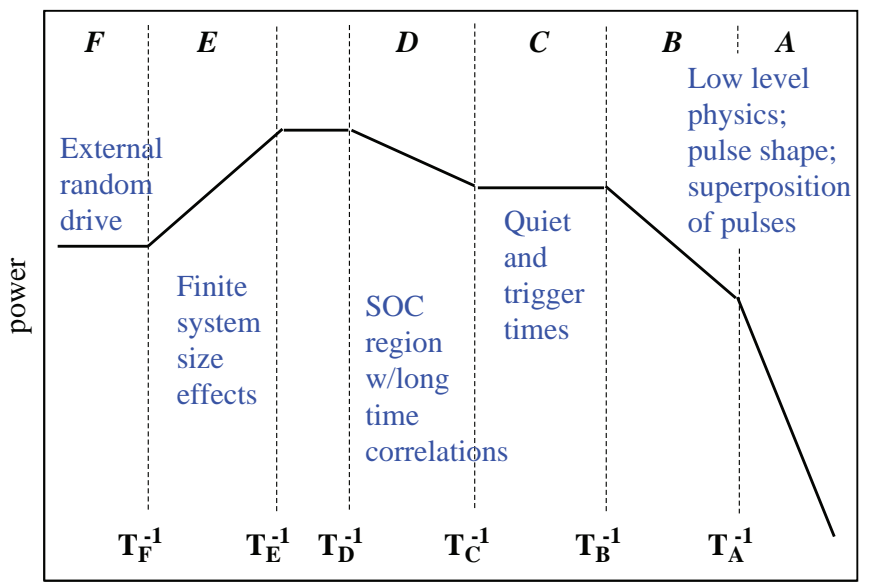

(a)

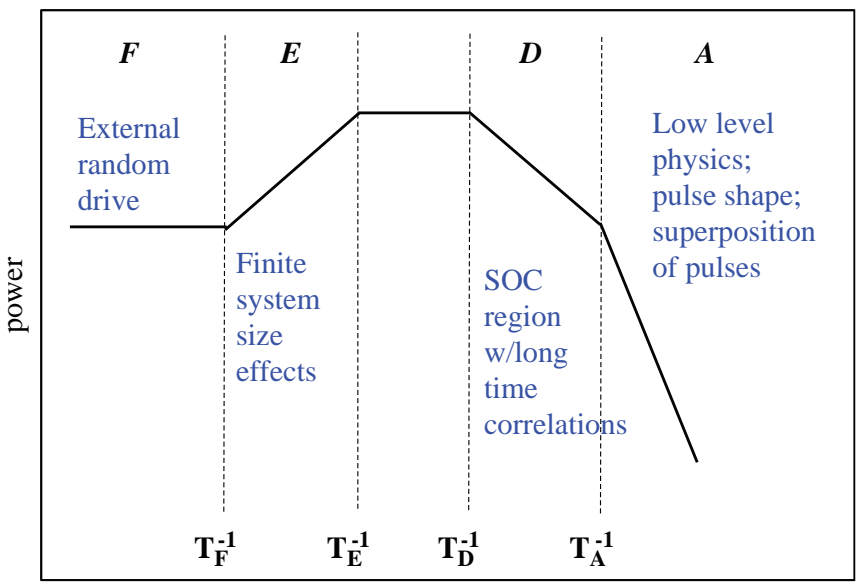

(c)

frequency

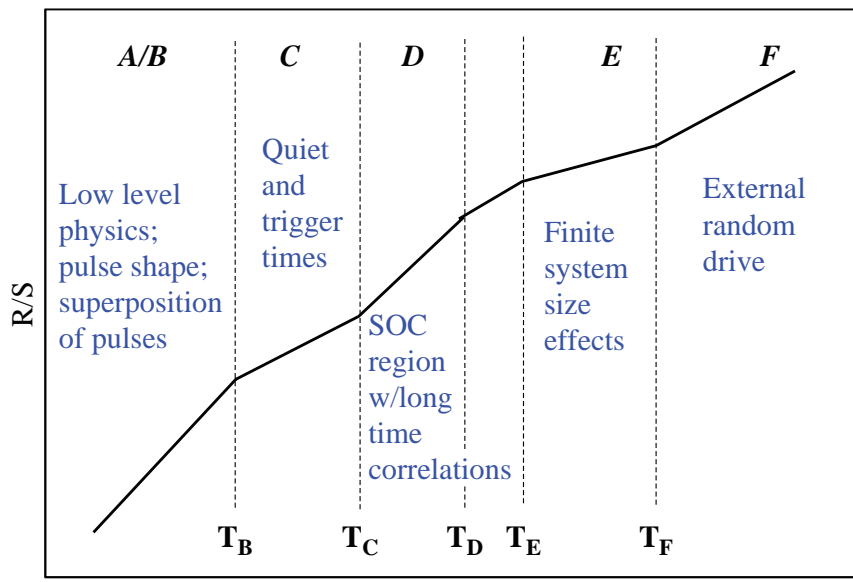

(b)

time lag

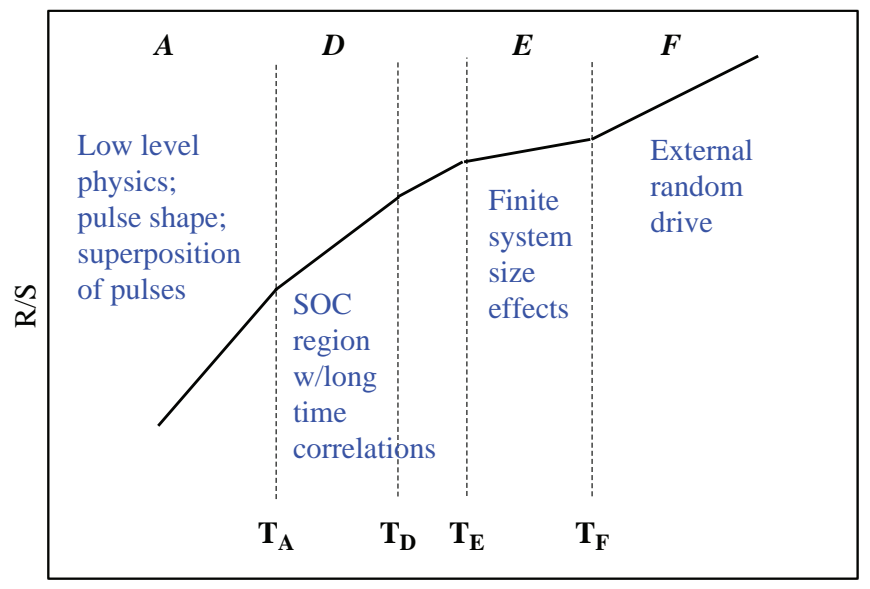

(d)

time lag

Fig. 4. Cartoons of distinct regions and their breakpoints and causes of power spectra and $R / S$ analysis of sandpile activity. (a) Power spectrum of low-drive, (b) $R / S$ analysis of low-drive, (c) power spectrum of high-drive and (d) $R / S$ analysis of high-drive. (c) is taken from Fig. 6 of Ref. [15] and the others are drawn in that spirit.

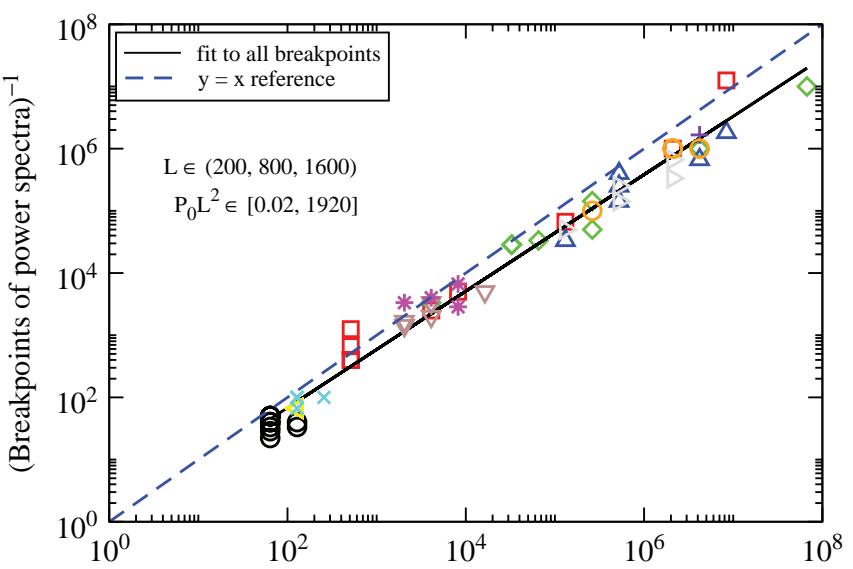

(a)

Breakpoints of R/S

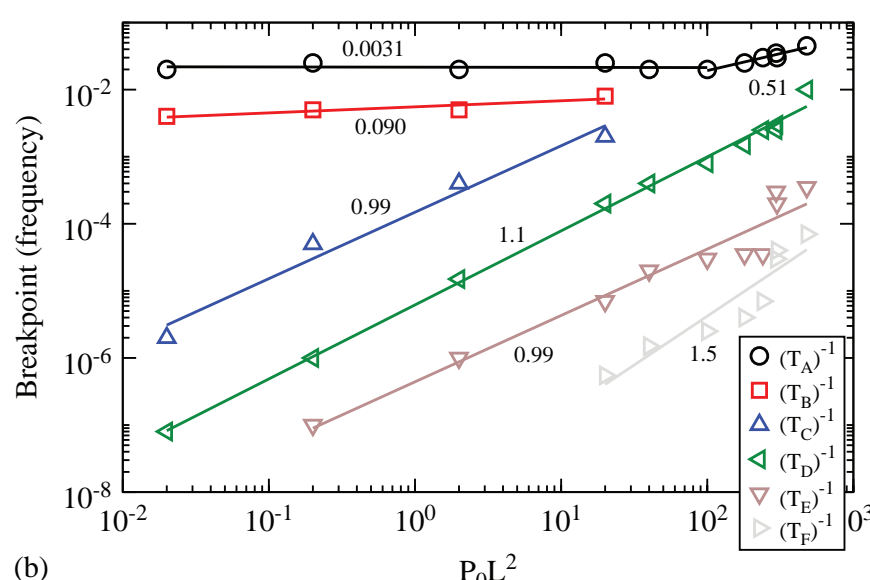

Fig. 5. (Color online) (a) Inverse breakpoints of the power spectra versus breakpoints of $R / S$ analysis of activity for different size sandpiles and driving rate and (b) breakpoints of power spectra versus driving rate for $L=200$ sandpile. Numbers shown are exponents of power law fits to the data. 

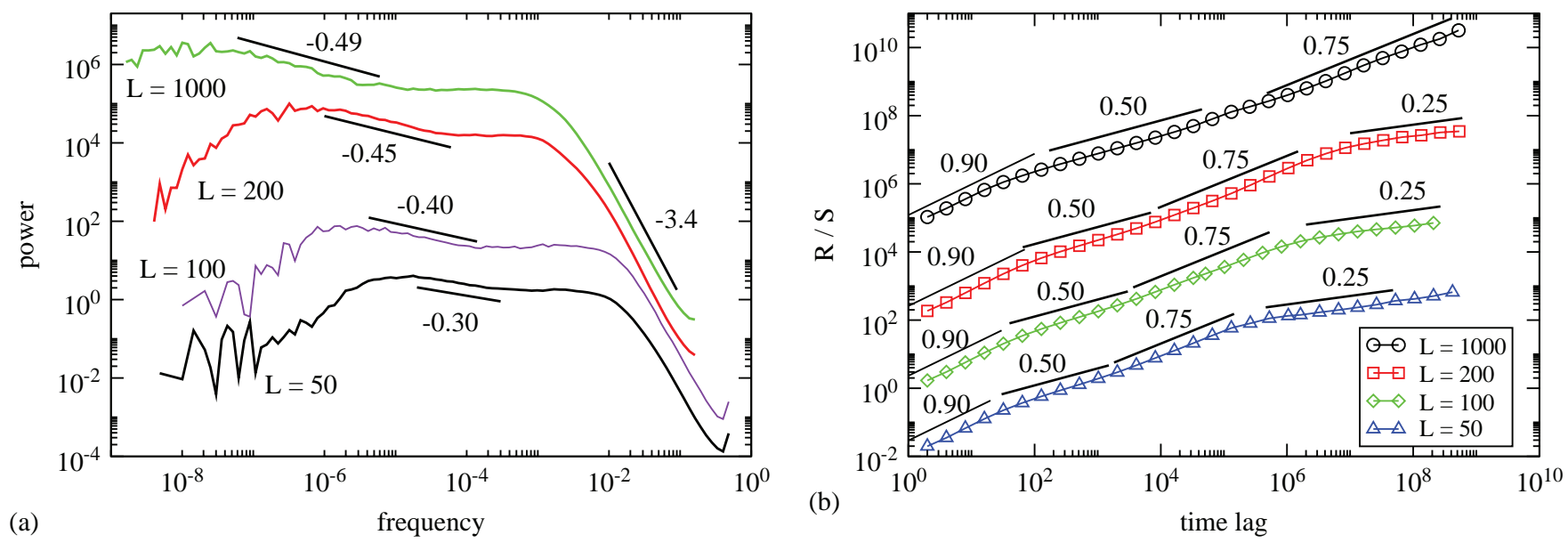

Fig. 6. Power spectra and $R / S$ analysis of activity for low-drive sandpile for $P_{0} L^{2}=0.2$ and $L=50,100,200,1000$. Plots have been shifted along the $y$ axes for better viewing. The solid lines are power laws plotted with the labeled exponent; they are not fits to the data. These plots show how the distinct regions move to longer time scales with larger system size but with the same effective driving rate, $P_{0} L^{2}$.

To conclude the enumeration of results, we will briefly describe the effect that changing the sandpile size has on the signatures and then more fully discuss this effect in Section 5. If $L$ is increased while $P_{0} L^{2}$ is held constant, then all regions move to smaller frequencies (or longer time lags), Fig. 6. The values of the exponents associated with each distinct region remain essentially unchanged, especially in the $R / S$ analysis.

\section{Discussion}

The first point that can be made from Figs. 2, 3 and 6 is that long time correlations do exist in the running sandpile regardless of driving rate. Changing the rate at which sand is dropped only changes the timescales over which the correlations are seen. This is evident in the spectra of Fig. 2, where the prominent hump, whose right side contains the $f^{-\beta}(0<\beta<1)$ correlated region, moves from low-frequency at low-drive to high frequency at high-drive. The fact that this hump exists at all is proof of correlations in the system, since a random superposition of pulses has a flat $f^{0}$ spectrum at low frequencies $[13,14]$. This is further confirmed by taking an activity time series from a low-drive case, where events are distinct and separated, and randomly shuffling the events to make a new time series. The spectrum and $R / S$ of the new time series show a $f^{0}$ and $H=0.5$ region, respectively, indicating no correlations, whereas before both measures showed correlated dynamics (Fig. 7). Note that this simple experiment also tells us that it is the avalanche order in which correlations are present, since the order of the quiet times among them merely reflects the random character of the drive [6]. Therefore, the previously mentioned notions that "strong correlations between successive [events is] at variance with the SOC model" [3] and that an event "can occur randomly anywhere at any time and ... cannot "know" how large it will become" [7,9] are misleading.

Before separately discussing the physical meaning of the different regions, it is interesting to ask ourselves why this hump and its associated correlated region has not been discussed in the past, giving rise to the aforementioned confusion. The only explanation we can offer is that the time series examined were simply too short, thus missing the timescales where correlations exist. It is instructive to note that in Fig. 4 of Ref. [15], the power spectrum of the low-drive case exhibits only regions $A, B$ and $C$, while the spectrum for the highdrive case barely extends to the beginning of region $E$. With that limited amount of dynamical information it is reasonable that the existence of dynamical correlations was mistaken for a consequence of the onset of avalanche overlapping. Proper credit, however, must indeed be given to the authors of Ref. [15] for their correct interpretation of the physical meaning of regions $A, B$ and $E$. For that reason, we will just briefly discuss them in what follows, instead emphasizing the interpretation of regions $C, D$ (the one that carries the signature of long time correlations) and $F$. 

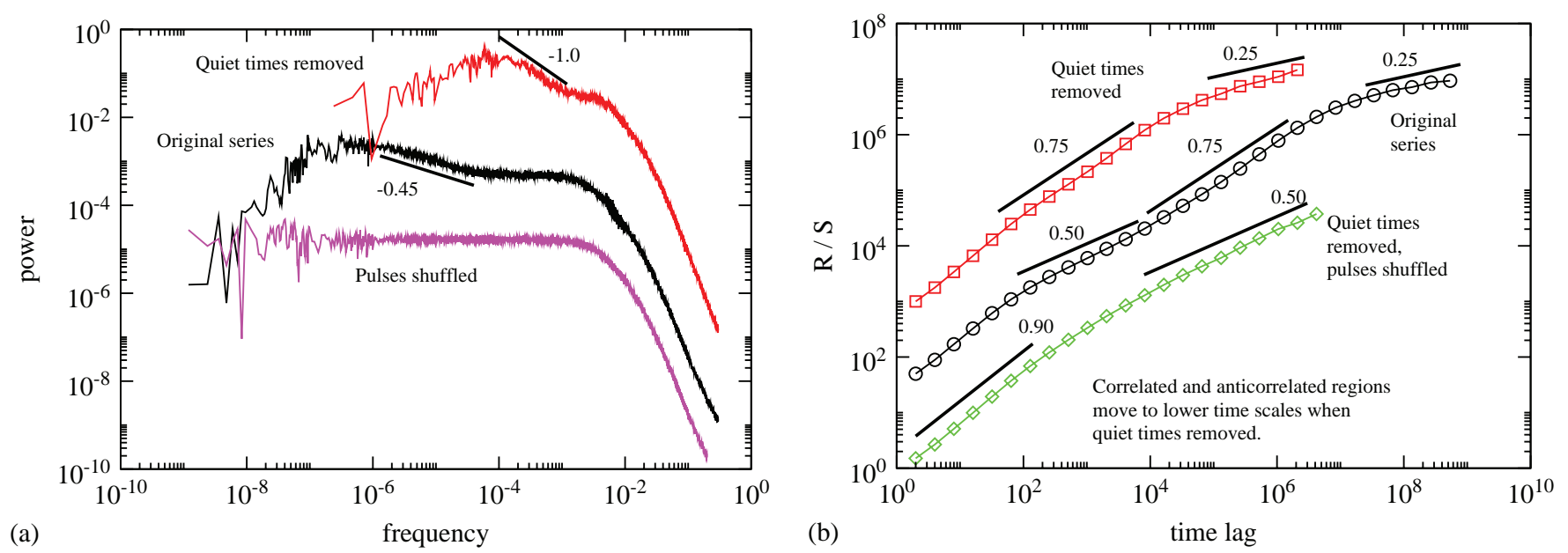

Fig. 7. Power spectra and $R / S$ analysis of low-drive sandpile: original series, with quiet times removed and with pulses shuffled. Plots have been shifted along the $y$ axes for better viewing. The solid lines are power laws plotted with the labeled exponent; they are not fits to the data. The spectrum and $R / S$ of the activity time series for the zero-drive sandpile are virtually identical to the spectrum and $R / S$ of the low-drive series with quiet times removed; to preserve clarity, they are not shown.

\subsection{Regions $A$ and $B$ (and $A / B)$ : pulse shapes}

Regions $A$ and $B$ at low-drive and region $A$ at high-drive are due to the low-level physics of the system that is manifested as the trapezoid pulse shape of individual avalanches (see Fig. 1). The values of the slopes and the location of the breakpoints of these regions can in fact be predicted by simply assuming a random superposition of trapezoids, in an analogous way to that which was originally done in Refs. [13,14] and noted for the high-drive case in Ref. [15]. The reason why this approach works is that regions $A$ and $B$ do not depend on the character of the system dynamics. There are two high-frequency regions $(A$ and $B)$ in the sandpile spectrum as opposed to the single one derived in Refs. [13,14] because those studies assumed a rectangular pulse shape. Such pulses have a single spectral region while trapezoids have two. Simple tests show that replacing the trapezoids in the sandpile activity time series by either rectangular pulses or by spikes ("delta" functions) results in appropriate changes to the exponents in regions $A$ and $B$ but in no change to regions $C-E$. The $R / S$ analysis exhibits only one region $(A / B)$ that ends at a breakpoint that coincides with $T_{\mathrm{B}}^{-1}$. Therefore, regions $A$ and $B$ of the power spectrum and region $A / B$ of the $R / S$ analysis correspond to the same low-level physics of the system. In fact, note that $H \simeq 1$ in region $A / B$, which implies that the value of the breakpoint $T_{\mathrm{B}}$ is related to the time series autocorrelation time and thus to the average avalanche duration.

Therefore, it is clear that the value of the exponents found in regions $A$ and $B$ does not have a dynamical origin as has been pointed out previously [13-15]. This can be easily proved by shuffling the time series: keeping the trapezoids intact but placing them randomly in time. This reordering necessarily makes all dynamical correlations vanish. It is reflected in the spectra by the disappearance of regions $D$ and $E$ and the lack of change of regions $A$ and $B$ (see Fig. 7). This is an important statement of the lowest level physics of the system. It tells us that the high-frequency part of the spectra of systems with different low-level physics and different fundamental pulse shapes may scale differently even if they exhibit the same low-frequency part of the spectrum because they share the same kind of dynamics.

\subsection{Region C: quiet times}

This is a region not previously identified in the running sandpile. In contrast to the vanishing drive limit, quiet times (periods with no activity) between avalanches appear in the running sandpile $[5,6]$. In essence, quiet times are a measure of the time that the system spends in an absorbing state. They are the source of the appearance of region $C$, which scales as $f^{0}$ in the power spectrum and as $H \simeq 0.5$ in the $R / S$ analysis, both being signatures of an uncorrelated Poisson process. These exponents reflect the random character of the 
external drive that causes avalanches to be randomly triggered. Besides SOC models, quiet times have been studied in other physical systems $[8,35]$.

Beyond the timescale of the largest single avalanche, $T_{\mathrm{B}} \sim L$, there can be a period where the correlations are dominated by the random triggering. The width of region $C$ is inversely related to the driving rate for a fixed system size. In fact, it disappears in the higher drive cases, after the average quiet time becomes on the order of the characteristic avalanche duration, $T_{\mathrm{B}}$. Also, the lower the driving rate $P_{0} L$, the fewer avalanches that occur in a given time period and the longer the system must "wait" for enough avalanches to occur to correlate with each other. The cutoff, $T_{\mathrm{C}}$, is a measure of this approximate minimum time and it scales inversely with the system time scale, $\sim P_{0}^{-1}$.

Note that quiet times do not have a physical meaning in the zero-drive limit of any sandpile, when the addition of sand is suspended during an active avalanche and is reinitiated immediately after it is completed. Their presence in the running sandpile does not change the dynamics, but allows the external drive to set a physical measure of time, $P_{0}^{-1}$, and to estimate the average quiet time [6]. The test shown in Fig. 7 justifies this statement: the spectrum and $R / S$ analysis of the zero-drive case are identical with those of the low-drive case with quiet times removed. Note that a low-drive case must be used for this exercise, so that there are few or no overlapping avalanches. We emphasize this to introduce the next section, which shows that overlapping of events is not necessary to produce correlated dynamics.

\subsection{Region D: dynamical correlations}

Proving the existence of and understanding the physics of region $D$ is the main point of this paper. For all cases in the low-drive regime, regardless of system size, we find $H \simeq 0.8$ in region $D$, indicating long time correlations. For the same timescale range, an $f^{-\beta}$ region with $0<\beta<1$ is found in the power spectra, again a signature of correlations. While $H$ remains constant, though, $\beta$ changes with driving rate (see Fig. 8): it approaches 1 at the highest drives and decreases as drive strength is reduced.

It is easy to show that the correlations must arise from interactions among distinct events and not from overlapping of events. The first clue is that this region exists on timescales far greater than the maximum duration of an individual avalanche $\left(T_{\mathrm{B}}\right)$. Since the activity time series consists of a succession of avalanches separated by quiet times and there is no (or very little) overlapping in the low-drive regime, the correlations must be due to the specific order of events, chosen by the system dynamics in spite of the randomness of the drive. This is confirmed by the random shuffling of the activity series mentioned when discussing regions $A$ and $B$ (see Fig. 7). Regions $C-F$ collapse into a single uncorrelated region for all time scales beyond $T_{\mathrm{B}}$, where

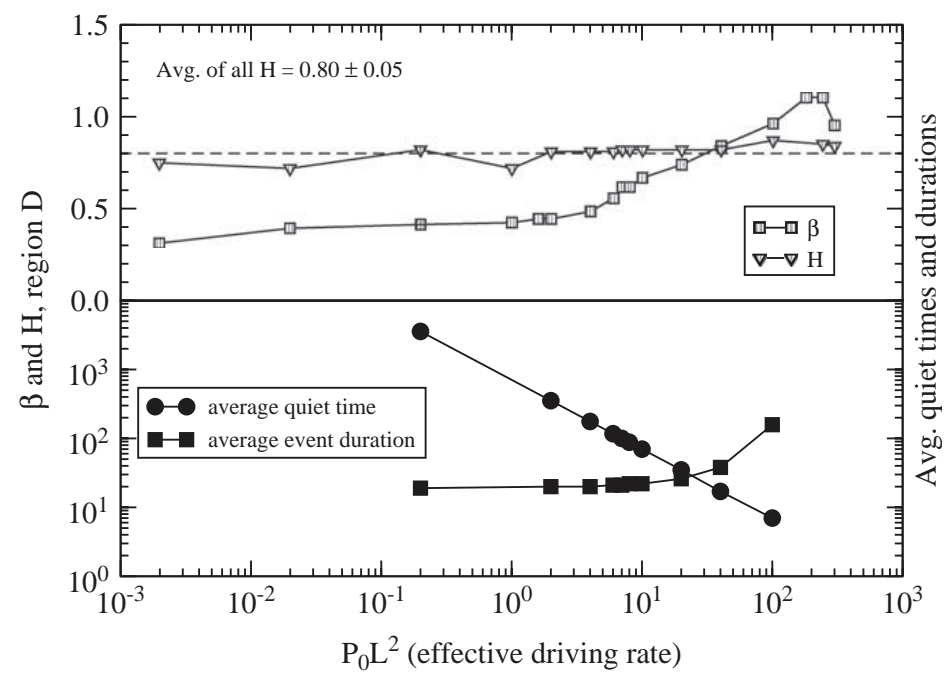

Fig. 8. $H, \beta$, average quiet time and average duration versus five orders of magnitude of effective driving rate. $H$ stays constant with changing driving rate while $\beta$ increases as driving rate increases, saturating at around $\beta=1$. 
the shuffled series has a $f^{0}$ spectrum down to the lowest frequencies and a Hurst exponent $H \simeq 0.5$ to the longest time scales.

A second confirmation that the correlations are established among the separate events is that quiet times have no role in the correlations. This is confirmed by the test already mentioned when discussing region $C$ : removing all quiet times from the activity data while keeping the event order unchanged. As seen in Fig. 7 , region $C$ disappears: the $H \simeq 0.5$ region is replaced by a $H \simeq 0.8$ region, which means that the $H \simeq 0.8$ region $D$ of the original data has moved to shorter timescales due to the removal of quiet times and the concomitant shortening of the series. But the correlations among events remain the same since neither their order nor their sizes have been changed.

The same value of $H \simeq 0.8$ is found for this region regardless of system size or driving rate, even in the vanishing drive limit in which SOC is strictly defined [17]. This simple fact suggests that the correlations present at the SOC critical point do persist almost without distortion at finite drives. In fact, this is still the case even at the highest drives, when avalanches do overlap and form superpulses. A superpulse is defined as the structure between successive quiet times in a high-drive activity time series (Fig. 1(b)). Note that quiet times in the high-drive cases are very small, on average. By randomly shuffling the superpulses of a high-drive time series, we see that the correlations that lead to $H \simeq 0.8$ are on time scales shorter than the average superpulse. Beyond these time scales, $\beta \simeq 0$ and $H \simeq 0.5$, signatures of uncorrelated data (Fig. 9).

Understanding the power spectrum of region $D$ is not as simple as for the $R / S$ analysis. The slope of this region, $\beta$, changes with driving rate and with system size. $1 / f$ regions are only obtained for high-drives (or by removing the quiet times of low-drive cases, which makes the system approach the zero-drive limit as previously discussed). This implies that a $1 / f$ region is not a necessary signature of SOC dynamics at finite drives, since correlations are still present, as indicated by the Hurst exponent remaining constant at $H \simeq 0.8$. In this sense, $R / S$ analysis is a more robust measure of the degree of correlations among events than is the power spectrum.

The question, however, remains open about why $\beta$ changes as region $D$ is pushed towards different timescales, and why $\beta$ saturates at 1 and not another value. We do not know the answer, but we can say a couple of things. First, it appears that $1 / f$ is due more to a lack of quiet times and distinct pulse shapes than to overlapping, at least in the sandpile model. We showed in Fig. 7 that removing all quiet times from between separate events of a low-drive activity time series produces a spectrum with a region $D$ where $\beta=1$. In that case, there is no overlapping of events by design. We can also discard the need for hidden additional correlations being established dynamically, since the same change in $\beta$ is observed when inserting randomlydistributed quiet times into an artificial time series of fractional Gaussian noise ( $f \mathrm{Gn}$ ) [29] with an arbitrary Hurst exponent $H=0.8$ (see Fig. 10). We inserted quiet times with an average approximately the same as the average of the original $f \mathrm{Gn}$ series $(\simeq 20)$. The spectrum and $R / S$ analysis are shown for the original and modified series. The original fGn series follows $\beta=2 H-1$, with $H \simeq 0.8$ and $\beta \simeq 0.6$. When quiet times are
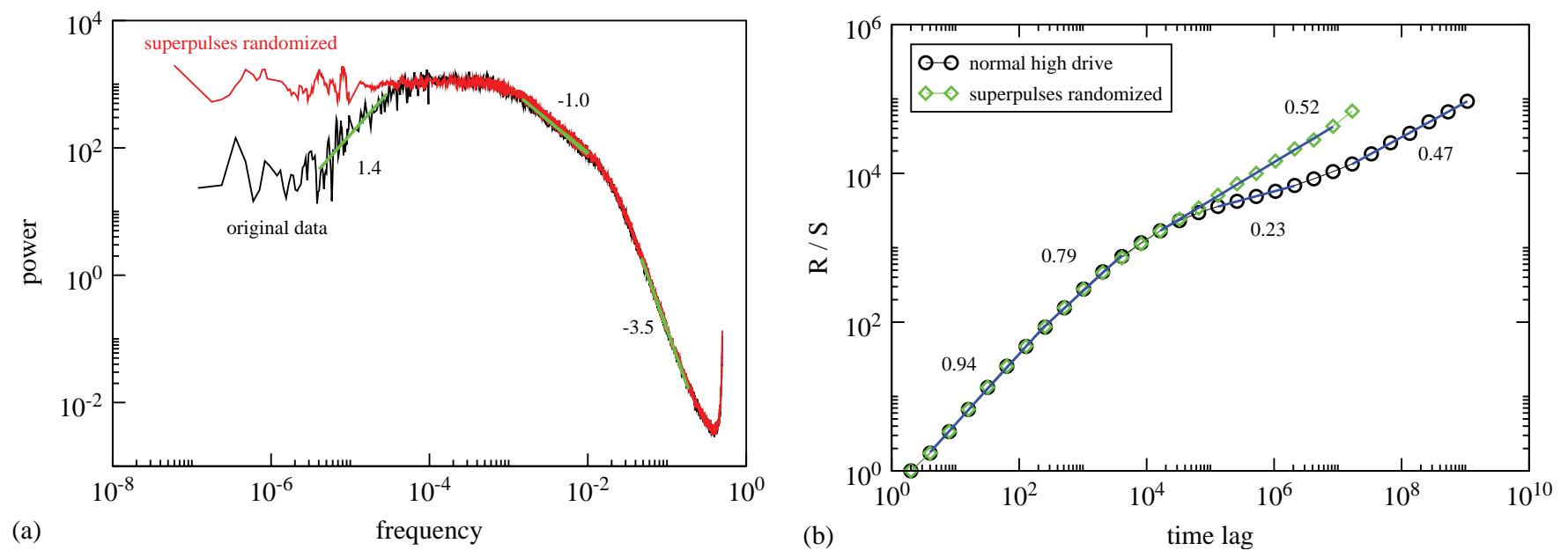

Fig. 9. (Color online) Power spectra and $R / S$ for high-drive with superpulses changed. Numbers shown are $\beta$ for spectra and $H$ for $R / S$. 


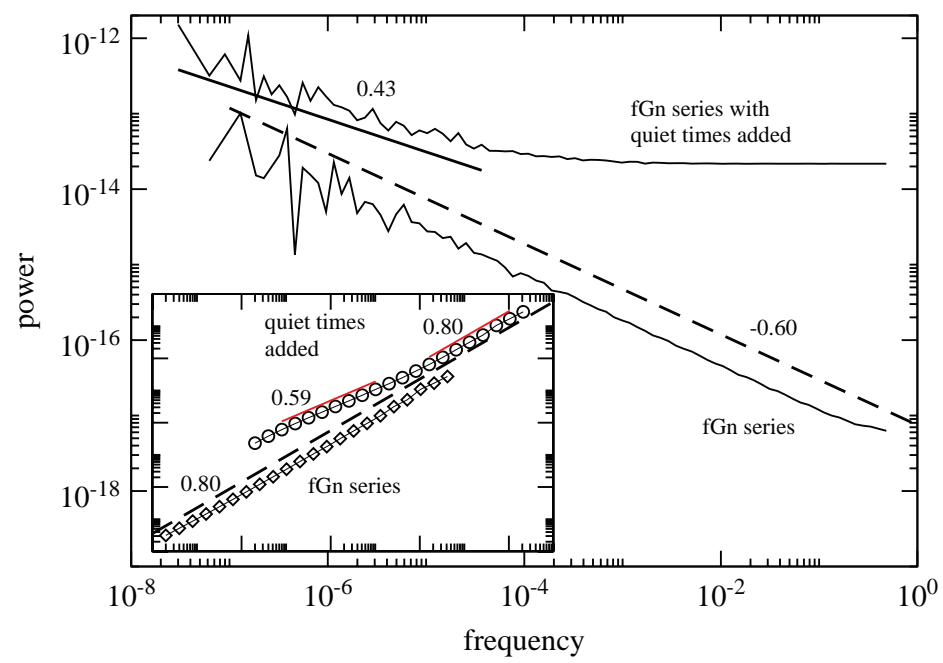

Fig. 10. Power spectra and (inset) $R / S$ analysis for fractional Gaussian noise of $H=0.8$ with and without quiet times added. Numbers shown are $\beta$ for spectra and $H$ for $R / S$.

added between each point of the original series a region of $H \simeq 0.5$ appears up to a certain time lag. Beyond this time lag, $H \simeq 0.8$ as expected since the correlations among the data have not changed. In the spectrum, $\beta \simeq 0$ down to the inverse of the same time lag and then $\beta \simeq 0.43$. This constant $H$ and changing $\beta$ is the same effect seen in the sandpile data.

\subsection{Region E: global discharge region}

Region $E$ is the discharge event region, already well-studied by Ref. [15] for high-drive, and a consequence of the finite system size. The finite sandpile is always being driven towards a globally critical slope. Once all or almost all cells have slope $\geqslant Z_{\text {crit }}-N_{\mathrm{f}}$, a keystone toppling, usually near the bottom, will produce a systemsize avalanche or a rapid succession of smaller avalanches that removes enough sand so that the slopes at all sites are reduced to much less than critical $\left(<Z_{\text {crit }}-N_{\mathrm{f}}\right)$. Once such a large event occurs, it is unlikely that one will happen again for a long time. Hence, large events are anticorrelated and have the signatures of anticorrelated dynamics, $\beta<0$ in the spectrum and $H<0.5$ in $R / S$ analysis. The same process drives both the low and high-drive cases.

\subsection{Region F: beyond dynamics}

Beyond breakpoint $T_{\mathrm{F}}^{-1}$, at the lowest frequencies, the spectrum is flat, which we conjecture to be a reflection of the random drive of the system on the longest time scales. Note that all dynamics is on time scales much smaller than those in this region. Correlations are eventually erased by the system-wide events and shuffled by the random drive at time scales longer than $T_{\mathrm{F}}$. Region $F$ extends to infinitely low frequencies; there are no further dynamical regions below it.

\section{Relation between system size and driving rate}

We return to Fig. 6 to discuss the effect of changing system size. It is reasonable to increase the level of forcing - practically, the amount of avalanche overlap — in the system by increasing the driving rate $P_{0}$. This means that more grains per time step will fall on the sandpile and triggering time will be reduced. It turns out that keeping $P_{0}$ fixed while increasing system size $L$ has the same effect because avalanches last longer in the larger system. In both of these cases, $P_{0} L$ has increased, causing trigger time to decrease. But since system size $L$ has remained constant, the ratio of average avalanche duration to trigger time has increased further, producing more overlap and stronger effective forcing. 
But the combination $P_{0} L$ does not, by itself, give enough information about the character of the drive as represented by the amount of avalanche overlap. Imagine increasing $L$ while decreasing $P_{0}$ so that $P_{0} L$ and, therefore, average trigger time remain constant. Though trigger time remains constant, the ratio of average avalanche duration to trigger time has again increased because of the increase in system size. This process, then, also effectively increases the driving rate in the larger system by producing more overlap among the individual avalanches that now last longer. This is true even though the original and the larger system both have the same $P_{0} L$ and, therefore, the same flux into the whole system and out of the bottom of the sandpile.

In terms of the effective driving rate discussed in Section $2, J_{\mathrm{E}}$ is larger for the larger system when $P_{0} L$ is the same in both. This feature can be exploited to produce a plot qualitatively similar to Fig. 2. In that figure, the driving rate $P_{0} L$ of the sandpile increases from top to bottom for the spectra shown while system size $L$ remains constant. If instead, one started with the spectrum of a small system at the top of the figure and increased system size while keeping $P_{0} L$ constant, then the same figure would be obtained. In both cases, $P_{0} L^{2}$ increases from top to bottom. An application of this is that perhaps one wants to study a physical system that is suspected of being described by SOC and/or the running sandpile. If experimenters cannot change the system size (like, for instance, that of the magnetosphere) then perhaps periods of different observed driving rates could be used as a proxy for different system sizes.

Since $J_{\mathrm{E}}$ is the correct quantity to use to compare systems in the same drive regime but where the individual parameters are different, this has implications, for example, in investigations of finite size and/or multifractal scaling. As an illustration, Fig. 11 shows justification for comparing systems with the same effective driving rate, in this case with $P_{0} L^{2}=2.0$. The spectra of systems with different driving rates $P_{0} L$ and system sizes $L$ but with the same $P_{0} L^{2}$ can be rescaled to lie on top of each other. The spectra of systems with the same driving rate $P_{0} L$ but with different system size $L$ cannot be rescaled to lie on top of each other.

Finally, we show in Fig. 12 the PDFs of avalanche durations from two different system sizes that have been rescaled by a factor of $L^{-\alpha}$ with $\alpha=0.34$. The rescaling has been done for a medium driving rate of $P_{0} L^{2}=2.0$ so that there is some avalanche overlapping. This, coupled with the rescaling of the spectra, shows that the running sandpile exhibits critical dynamics similar to that seen in the sandpile with vanishing drive [25].

\section{Conclusions}

We have analyzed the one-dimensional directed running sandpile model for five-orders of magnitude of driving rate and for almost three orders of magnitude of system size and have shown that the same dynamical correlations that are present in the SOC zero-drive limit persist (and produce non-trivial signatures in the

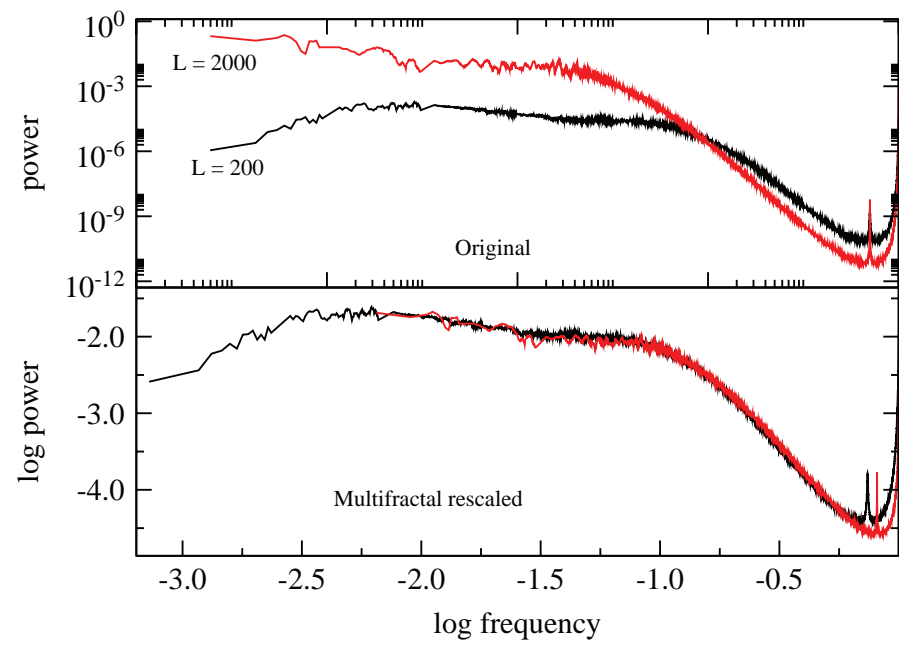

Fig. 11. (Color online) Rescaling of power spectra of two different systems with $P_{0} L^{2}=2.0$, one with $L=200$ and one with $L=2000$, using the rescaling law $y_{0} \log _{10} S(f)=g\left(x_{0} \log _{10}\left(f / x_{1}\right)\right)$, where $S(f)$ is the power spectrum and $g(f)$ is a scaling function. Different sets of parameters $\left[x_{0}, x_{1}, y_{0}\right]$ are needed for each spectrum so this is not the same as, for instance, the multifractal rescaling of avalanche size PDFs of Ref. [25], where the same value of each parameter is used for different system sizes. 


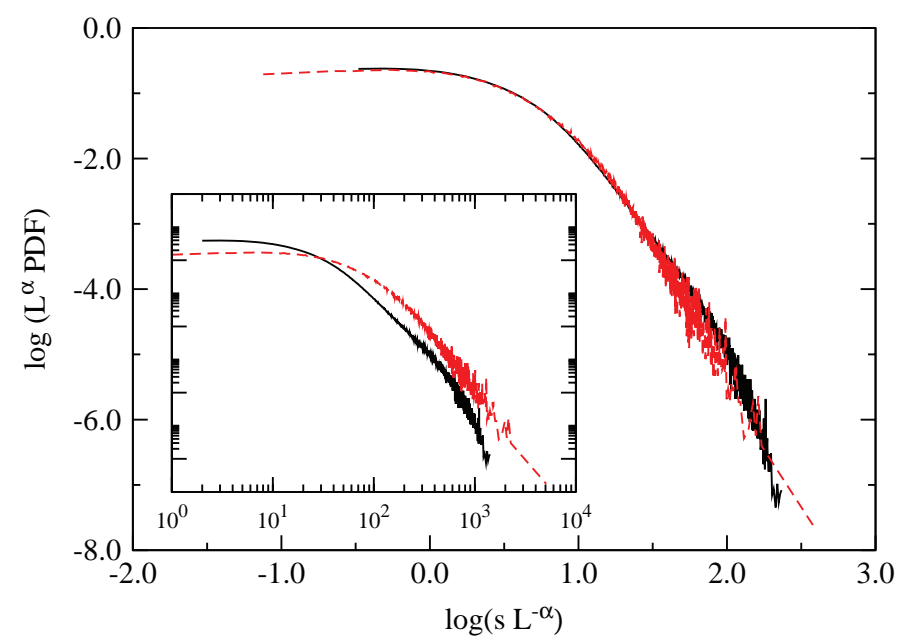

Fig. 12. (Color online) Rescaling of PDF of avalanche durations for two system sizes, $L=200$ (solid line) and $L=2000$ (dashed line), and a shared effective driving rate $P_{0} L^{2}=2.0$. The PDFs have been rescaled by $L^{\alpha}$ where $\alpha=0.34$.

power spectra and we show through rescaling of power spectra and avalanche size PDFs that the running sandpile exhibits SOC in the same way that the vanishing drive sandpile does. $R / S$ analysis of activity time series) at finite drives. Our results warn that a time series from any system, for which SOC may provide a plausible description of the dynamics, may be too short to see these correlation signatures and could thus be mistaken for a simple random time series. We show through rescaling of power spectra and avalanche size PDFs that the running sandpile exhibits SOC in the same way that the vanishing drive sandpile does.

The reason why these correlations persist away from the SOC critical point at zero-drive can be understood when discussing the physical mechanism through which the sandpile establishes dynamical correlations. It is well known that memory in the sandpile is retained in the heights (and, therefore, the local gradients) of each cell. This is, for a physical system, the instantaneous system state. When an avalanche ends, the cells at the endpoints of its active zone are closer to critical and are more likely to be the initiation point of another avalanche. This is the basis for memory in the system and explains why the drive strength does not affect dynamical correlations, except for pushing them to longer or shorter time scales: regardless of how long the intervening quiet time is between two events, they are correlated in the same way. In reference to the introduction, this is how an event can 'know' how large it will become - it can grow only as large as the size of the local neighborhood of marginal cells. Also, this is why an event of any size cannot simply occur at any time or place in the system. Only the time of the triggering of an event is random due to the random external forcing. Before that trigger, though, the size and location of the event is predetermined by the state of the local neighborhood, like an earthquake fault ready to slip. Therefore, the same memory mechanism that is the basis for SOC at zero-drive is still present in the gradients regardless of how slowly or quickly sand is added, as long as the system is not overdriven.

As we have shown, region $D$ is the only region that reflects the underlying zero-drive SOC dynamics at finite drive: on the time scales in this region, the spectral and $R / S$ signatures reflect only long time correlations and nothing about pulse shape, quiet times, random superpositions, overlapping of pulses or system size. In fact, in the thermodynamic limit in which the system size becomes increasingly large, we must simultaneously allow $P_{0} \rightarrow 0$ to keep $J_{\mathrm{E}}=P_{0} L^{2}$ constant, which pushes the system to the SOC critical point [17-19]. In that case, region $D$ would extend to lower and lower frequencies.

Finally, another interesting result derived from this work is that the relation $\beta=2 H-1$ derived in Ref. [24] for $f \mathrm{Gn}$ series and often accepted as the correct relationship between $\beta$ and $H$ regardless of the system, does not hold in general. While this fact is known from general theory [24,30], the sandpile results provide a concrete example where the relation does not hold. This implies that the random drive $P_{0}$ of the system for which $\beta=2 H-1$ does hold is filtered by the sandpile so that $\beta$ and $H$ no longer have such a simple relation. Note that the changing $\beta$ and constant $H$ of the correlated region $D$ imply that the mechanism that produces 
$1 / f$ at the high-drive produces non- $1 / f$ at lower drive, so that " $1 / f$ is not always $1 / f$ " and smaller values of $\beta$ are not necessarily any less "special" than $\beta=1$.

\section{Acknowledgments}

Many thanks to H.J. Fletcher, N.W. Watkins and B.T. Werner for support and suggestions. Support from DOE under Grants DE-FG03-99ER54551 and DE-FG03-00ER54599 (a young investigator award) and NSF under Grant ECS-0085647 are gratefully acknowledged.

\section{References}

[1] P. Bak, C. Tang, K. Wiesenfeld, Self-organized criticality: an explanation of $1 / f$ noise, Phys. Rev. Lett. 59 (1987) 381.

[2] P. Bak, C. Tang, K. Wiesenfeld, Self-organized criticality, Phys. Rev. A 38 (1) (1988) 364-374.

[3] G. Boffetta, V. Carbone, P. Giuliani, P. Veltri, A. Vulpiani, Power laws in solar flares: self-organized criticality or turbulence? Phys. Rev. Lett. 83 (22) (1999) 4662-4664.

[4] E. Spada, V. Carbone, R. Cavazzana, L. Fattorini, G. Regnoli, N. Vianello, V. Antoni, E. Martines, G. Serianni, M. Spolaore, L. Tramontin, Search of self-organized criticality processes in magnetically confined plasmas: hints from the reversed field pinched configuration, Phys. Rev. Lett. 86 (14) (2001) 3032-3035.

[5] R. Sánchez, D.E. Newman, B.A. Carreras, Waiting-time statistics of self-organized criticality systems, Phys. Rev. Lett. 88 (6) (2002) 068302.

[6] R. Sánchez, D.E. Newman, W. Ferenbaugh, B.A. Carreras, V.E. Lynch, B.P. van Milligen, Quiet-time statistics: a tool to probe the dynamics of self-organized-criticality systems from within the strong overlapping regime, Phys. Rev. E 66 (2002) 036124.

[7] Is the reliable prediction of individual earthquakes a realistic scientific goal? Multi-author debate on Nature web site, 1999. URL 〈http: //www. nature.com/nature/debates/earthquake/〉.

[8] R. Sanchez, B. van milligen, D. Newman, B. Carreras, Quiet-time statistics of electrostatic turbulent fluxes from the JET tokamak and the w7-as and tj-ii stellarators, Phys. Rev. Lett. 90 (18) (2003) 185005.

[9] X. Yang, S. Du, J. Ma, Do earthquakes exhibit self-organized criticality? Phys. Rev. Lett. 92 (2004) 228501.

[10] R. Woodard, D.E. Newman, R. Sánchez, B.A. Carreras, Comment on “Do earthquakes exhibit self-organized criticality?” Phys. Rev. Lett. 93 (24) (2004) 249801.

[11] X. Yang, S. Du, J. Ma, Comment on “Do earthquakes exhibit self-organized criticality?”-reply, Phys. Rev. Lett. 93 (24) (2004) 249802.

[12] K. Christensen, Z. Olami, Variation of the Gutenberg-Richter $b$ values and nontrivial temporal correlations in a spring-block model for earthquakes, J. Geophys. Res. 97 (B6) (1992) 8729-8735.

[13] H.J. Jensen, K. Christensen, H.C. Fogedby, $1 / f$ noise, distribution of lifetimes, and a pile of sand, Phys. Rev. B 40 (10) (1989) $7425-7427$.

[14] J. Kertész, L.B. Kiss, The noise spectrum in the model of self-organised criticality, J. Phys. A 23 (1990) L433-L440.

[15] T. Hwa, M. Kardar, Avalanches, hydrodynamics, and discharge events in models of sandpiles, Phys. Rev. A 45 (1992) 7002.

[16] J. Davidsen, M. Paczuski, $f^{-\alpha}$ noise from correlations between avalanches in self-organized criticality, Phys. Rev. E 66 (2002) 050101(R).

[17] R. Dickman, A. Vespignani, S. Zapperi, Self-organized criticality as an absorbing-state phase transition, Phys. Rev. E 57 (5) (1998) 5095-5105.

[18] R. Dickman, M. Munoz, A. Vespignani, S. Zapperi, Paths to self-organized criticality, Braz. J. Phys. 30 (1) (2000) $27-41$.

[19] A. Vespignani, R. Dickman, M. Munoz, S. Zapperi, Absorbing-state phase transitions in fixed-energy sandpiles, Phys. Rev. E 62 (4) (2000) 4564-4582.

[20] G. Pruessner, O. Peters, Self-organized criticality and absorbing states: lessons from the Ising model, Phys. Rev. E 73 (2006) 025106(R) URL 〈http: / /arxiv.org/abs / cond-mat/0411709).

[21] E.T. Lu, Avalanches in continuum driven dissipative systems, Phys. Rev. Lett. 74 (13) (1995) 2511-2514.

[22] D.E. Newman, B.A. Carreras, P.H. Diamond, T.S. Hahm, The dynamics of marginality and self-organized criticality as a paradigm for turbulent transport, Phys. Plasmas 3 (1996) 1858.

[23] H.E. Hurst, Long-term storage capacity of reservoirs, Trans. Am. Soc. Civ. Eng. 116 (1951) 770.

[24] B.B. Mandelbrot, Gaussian Self-affinity and Fractals, Springer, Berlin, 2002.

[25] L.P. Kadanoff, S.R. Nagel, L. Wu, S. Min Zhou, Scaling and universality in avalanches, Phys. Rev. A 39 (12) (1989) $6524-6537$.

[26] H. Hinrichsen, Non-equilibrium critical phenomena and phase transitions into absorbing states, Adv. Phys. 49 (7) (2000) 815-958.

[27] H.J. Jensen, Self-Organized Criticality: Emergent Complex Behavior in Physical and Biological Systems, Cambridge University Press, Cambridge, 1998.

[28] R.N. Bracewell, The Fourier Transform and Its Applications, third ed., McGraw-Hill, New York, 2000.

[29] B.B. Mandelbrot, Multifractals and 1/f Noise, Springer, Berlin, 1999.

[30] B.B. Mandelbrot, The Fractal Geometry of Nature, W.H. Freeman and Company, New York, 1982. 
[33] C.X. Yu, M. Gilmore, W. Peebles, T. Rhodes, Structure function analysis of long-range correlations in plasma turbulence, Phys. Plasmas 10 (7) (2003) 2772-2779.

[34] M. Gilmore, C.X. Yu, T. Rhodes, W. Peebles, Investiations of rescaled range analysis, the hurst exponent, and long-time correlations in plasma turbulence, Phys. Plasmas 9 (4) (2002) 1312-1317.

[35] O. Peters, C. Hertlein, K. Christensen, A complexity view of rainfall, Phys. Rev. Lett. 88 (1) (2002) 018701 URL/http:// arxiv.org/abs/cond-mat/0201468). 